\title{
AN ADAPTIVE INTEGRATION MODEL OF VECTOR POLYLINE TO DEM DATA BASED ON SPHERICAL DEGENERATE QUADTREE GRIDS
}

\author{
Xuesheng Zhao *, Jiaojiao Wang, Zhengyi Yuan, Yuan Gao \\ Geoscience and Surveying Engineering College, China University of Mining and Technology (Beijing), Beijing, China \\ - zxs@cumtb.edu.cn
}

KEY WORDS: Adaptive integration, DQG, DEM, Vector polyline, Shift algorithm

\begin{abstract}
:
Traditional geometry-based approach can maintain the characteristics of vector data. However, complex interpolation calculations limit its applications in high resolution and multi-source spatial data integration at spherical scale in digital earth systems. To overcome this deficiency, an adaptive integration model of vector polyline and spherical DEM is presented. Firstly, Degenerate Quadtree Grid (DQG) which is one of the partition models for global discrete grids, is selected as a basic framework for the adaptive integration model. Secondly, a novel shift algorithm is put forward based on DQG proximity search. The main idea of shift algorithm is that the vector node in a DQG cell moves to the cell corner-point when the displayed area of the cell is smaller or equal to a pixel of screen in order to find a new vector polyline approximate to the original one, which avoids lots of interpolation calculations and achieves seamless integration. Detailed operation steps are elaborated and the complexity of algorithm is analyzed. Thirdly, a prototype system has been developed by using VC++ language and OpenGL 3D API. ASTER GDEM data and DCW roads data sets of Jiangxi province in China are selected to evaluate the performance. The result shows that time consumption of shift algorithm decreased about $76 \%$ than that of geometry-based approach. Analysis on the mean shift error from different dimensions has been implemented. In the end, the conclusions and future works in the integration of vector data and DEM based on discrete global grids are also given.
\end{abstract}

\section{INTRODUCTION}

In geographical information systems (GIS) vector data is one of the important information to store, represent and manage spatial entities. DEM focuses on describing the elevation information of the spatial features and can be simulated by using spatial discrete grid. The adaptive integration of vector data and spherical DEM data becomes one of the important issues in the community of Digital Earth visualization and innovation. The existing methods for vector data adapting to DEM mainly include two kinds: texture-based approach and geometry-based approach [Bruneton and Neyret 2008]. Texture-based approach rasterizes vector data into texture and maps it onto DEM, including texture mapping algorithm [Bruneton and Neyret 2008; Döllner 2005] and shadow volume algorithm [Schneider et al. 2005; Schneider and Klein 2007; Yang et al. 2010; Vaaraniemi et al. 2011]. It is widely used in most digital earth systems such as World Wind and ArcGlobe due to its relatively high efficiency, but it is hard to perform multi-scale query, calculation and decision analysis and results in distortion when zooming in vector data. Geometry-based approach generates many new points to adapt vector data to terrain, including geometry-overlay algorithm [Szenberg et al. 1997; Agrawal et al. 2006] and geometry-embed algorithm [Schilling et al. 2008]. It maintains the original characteristics of vector data and takes advantage of secondary data to constrain DEM [Vaaraniemi et al. 2011], but lots of geometric interpolation calculation has become a bottleneck problem urgent to be solved [Schneider and Klein 2007; Sun et al. 2012]. Moreover, traditional geometry-based approach is limited to be mainly used in plane Euclidean space.
Compared with Equal Latitude-Longitude Grid, Polyhedron Subdivision Grid and Adaptive Subdivision Grid, Degenerate Quadtree Grid (DQG) has the advantages such as simple structure, radial symmetry, uniform orientation, translation congruence and data transferability, easy to execute the operation such as multi-level index, proximity search, coordinate conversion, the organization, management and compressed storage of multi-resolution data [Cui and Zhao 2007]. Focus on the complex interpolation calculation, low efficiency and its extension application at spherical grid scale of the geometry-based approach in this paper, an adaptive integration model of vector polyline and DEM based on spherical DQG is presented. The idea of DQG cell decomposition is introduced and a novel shift algorithm is developed and greatly improves the integration efficiency. The analysis and discuss on the integration model is given in the end.

\section{THE METHOD OF DQG SUBDIVISION AND CELL DECOMPOSITION}

The method of DQG subdivision is as below: Firstly, inscribed regular octahedron is selected as basis of sphere subdivision. Spherical surface will be divided into 8 regular spherical triangles. Secondly, for every spherical triangle, the midpoints of the 2 waist sides are connected to form a new latitude line. The midpoint of the bottom edge of spherical triangle and the midpoint of the new latitude line are connected to form a new longitude line. Thus, every spherical triangle can be divided into a new spherical triangle and 2 new spherical quadrilaterals, in which the spherical triangle will be tessellated according to

* Corresponding author. zxs@cumtb.edu.cn. 
the above steps and the spherical quadrilaterals will be subdivided according to conventional quadtree subdivision, and

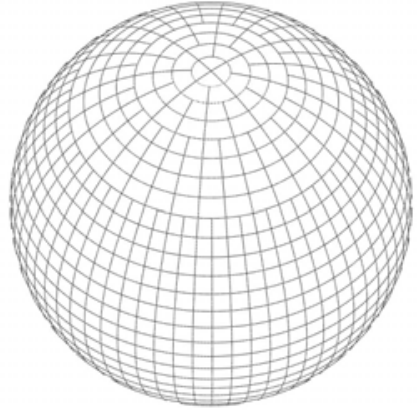

a. level 4

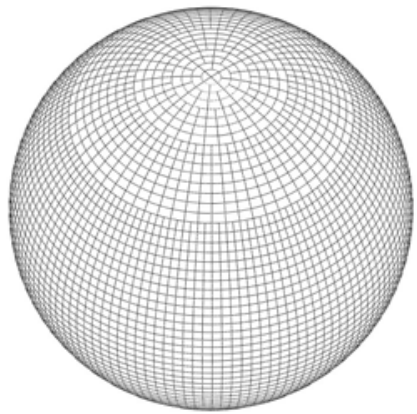

b. level 5

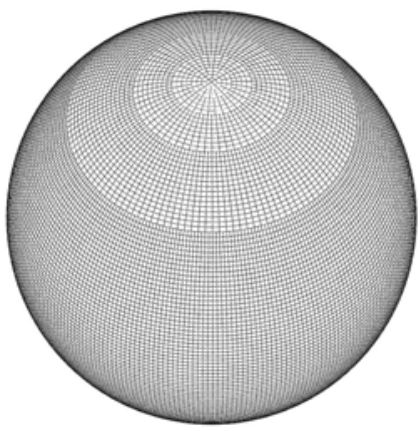

c. level 5

Figure 1. Hierarchical subdivision of spherical surface based on octahedron [Cui and Zhao 2007].
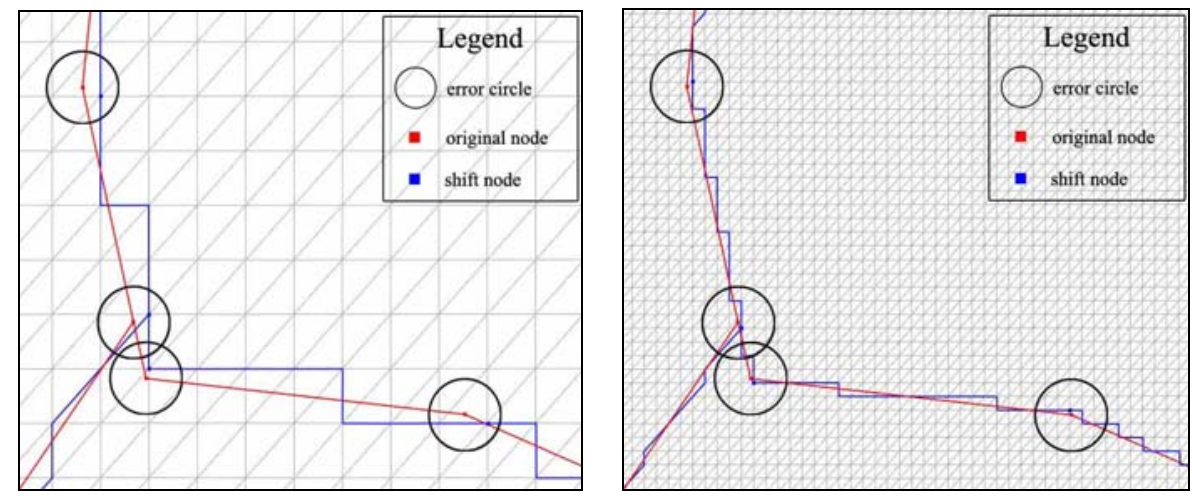

Figure 2. Shift operation and error circle based on different grid resolution (Left is level 16 and right is level 18)

The idea of cell decomposition[Zhou et al. 2009] includes three aspects:

(1) cell nodes stand for point features and accurate spatial location;

(2) cell boundaries measure the relationship among cell polygons or nodes;

(3) cell polygons stand for the polygon features.

This idea builds up the mapping and logic relationship between DQG cells and vector features, and is the important theoretical basis of the adaptive integration model. Based on cell decomposition, shift operation on the vector points would be done to meet the scale accuracy of map or the visual resolution of human being's eyes. In figure 2, the radius of error circle indicates the scale accuracy of the vector map, that is to say, the vector point shifting in the area of the circle would satisfy the measurement precision, and the original polyline after shift totally matches with the boundaries of DQG cell to achieve the seamless integration with spherical grid.

\section{AN SHIFT ALGORITHM}

\subsection{Basic principle of shift algorithm}

Firstly, the original polyline is divided into many segments every of which is made up of two adjacent nodes; for the DQG cells gone through by each segment, there is a new vector line approximate to the original one in the spatial distance and direction after shift operation on the vector nodes. In order to ensure the visual effects and meet the scale accuracy of map, shift operation should be done when the displacement distance is smaller or equal to the radius of error circle, otherwise, geometric interpolation calculation would be used.

Shift operation would be performed from the starting node to the end node of the original polyline until all of the nodes have been traversed. This approach is named 'shift algorithm' due to the frequent displacement of the vector points.

\subsection{Steps and flow of shift algorithm}

The detailed steps of shift algorithm mainly include the following:

Input: Spherical DEM based DQG at certain level and original nodes set $V=\left[\mathrm{v}_{0}, \mathrm{v}_{1}, \ldots, \mathrm{v}_{\mathrm{n}}\right]$.

Step1 Find the cell $G A$ where the starting point $A$ of $V$ lies;

Step2 Find the point $g a$ closest to $A$ in $G A$ and add $g a$ into the new nodes set $U$;

Step3 Shift operation on the other nodes of $V$, define $B$ is the point next to $A$;

Step3.1 IF $B$ lies in $G A$

Find the point $g b$ closest to $B$ in $G A$;

IF the line connected by $g b$ and the current end node $u k$ of $U$ goes through the diagonal, transition node $P$ would be determined according to the type of DQG cell:

Case one: for pole triangle cell, $P$ is one of the cell nodes (figure 3-1)

Case two: for quadtree cell, $P$ is the midpoint of diagonal (figure 3-2)

Case three: for degenerate quadtree cell, $P$ is the point closest to the line connected by $g b$ and $u k$ (figure $3-3,4,5$ ) 
Step3.2 IF $B$ does not lie in $G A$

Find the cell $G B$ where $B$ lies;

Find all the adjacent cell nodes of point $u k$ and define the set $K$;

Find the 4 or 8 neighbor node $k_{i}$ closest to $B$ (figure 4 ) and add it into $U$ :

IF $k_{i}$ does not lie in $G B$, find all the adjacent nodes of $k_{i}$;
Else $k_{i}$ falls in $G B$, find the node $g b$ in $G B$

closest to $B$ :

IF $g b \neq k_{i}$, add $g b$ into $U$;

Else eliminate $g b$.

Step3.3 Loop until the remaining nodes in $V$ have been completely traversed

Output: The nodes set $U=\left[u_{0}, u_{1}, \ldots, u_{m}\right]$ after shift

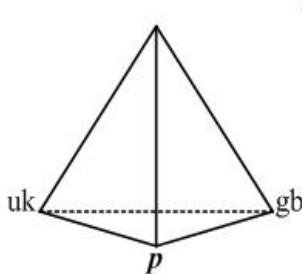

1

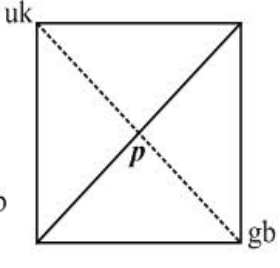

2

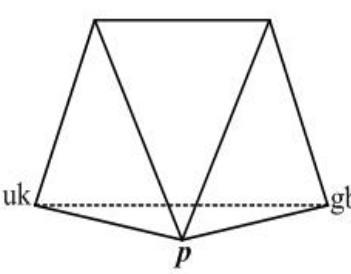

3
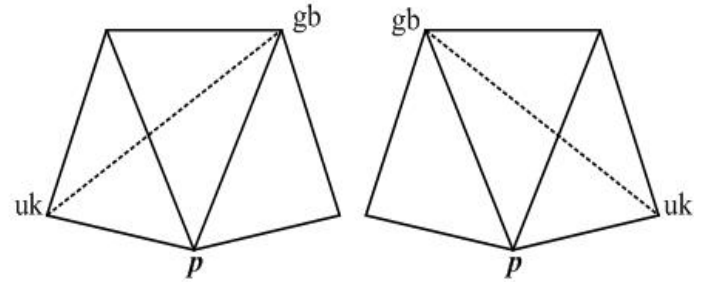

4

5

Figure 3. DQG pole triangle cell (1), quadtree cell (2), and degenerate quadtree cell (3-5).

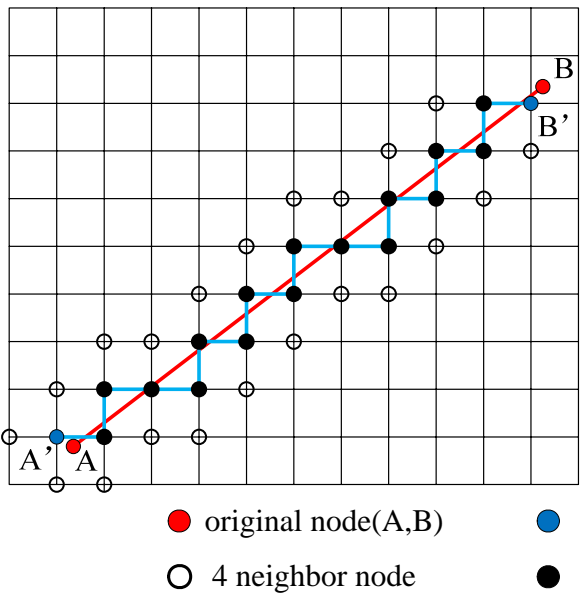

original polyline

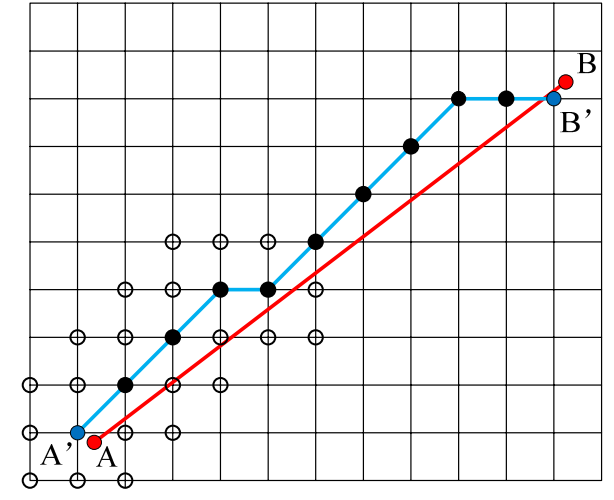

shift node(A' , B' )

4 or 8 neighbor node closest to B'

shift polyline

Figure 4. Shift operation based on 4 or 8 neighbor cells searching

\section{EXPERIMENTS AND DISCUSSIONS}

DEM data is from ASTER GDEM $(30 \mathrm{~m} \times 30 \mathrm{~m})$ distributed by NASA and METI. Vector data is road polyline in Jiangxi province of China from DCW(Digital Chart of the World) produced by Defense Mapping Agency with the scale $1: 1000,000$ and the size $161 \mathrm{~Kb}$. The prototype system for integration model is implemented in the context of Visual C++ and OpenGL API. The subdivision level of DQG in this experiment is up to 18 corresponding to the resolution of ASTER GDEM.

\subsection{Representation on the seamless integration model}

In this experiment, subdivision level 13-18 was selected for comparison. Full view of China road polyline on DQG DEM at level 11 before and after drift operation is shown as figure 5 . Figure 6 shows full view of Jiangxi roads polyline on DEM at level 13 before and after shift operation. Figure 7 shows near viewpoint of the representation that the roads polylines after shift operation correctly match with the terrain (figure 7-e,f,g), eliminating the phenomenon of suspension and penetration (figure 7-a,b,c) when original roads overlay onto the terrain before shift operation.

\subsection{Efficiency and error analysis on adaptive integration model}

Time consumption and the mean shift error is used to evaluate the performance of shift algorithm for adaptive integration model. The error generated by shift operation is calculated as following:

$$
\text { Error }=\frac{1}{N} \sum_{i}^{N}\left|C_{i}-\overline{C_{i}}\right|
$$

In equation (1), $N$ denotes the number of original points, $C_{i}$ and $\overline{C_{i}}$ respectively denotes 3-D coordinate of the point $(x, y, z)$ before and after shift operation. $\left|C_{i}-\overline{C_{i}}\right|$ denotes spherical distance between the original point and the new one obtained by shift operation.

Table 1 and figure 8 shows that time consumption for shift algorithm reduced about $76 \%$ of that for geometric interpolation 
International Archives of the Photogrammetry, Remote Sensing and Spatial Information Sciences, Volume XL-4/W2, 2013 ISPRS WebMGS 2013 \& DMGIS 2013, 11 - 12 November 2013, Xuzhou, Jiangsu, China

Topics: Global Spatial Grid \& Cloud-based Services

at level 13-18 and increased much more slowly than that for geometric interpolation with spatial resolution rising. The mean error from shift operation decreased with the level increasing, while the ratio of mean error to average edge length of DQG cell was about $1 / 2$.
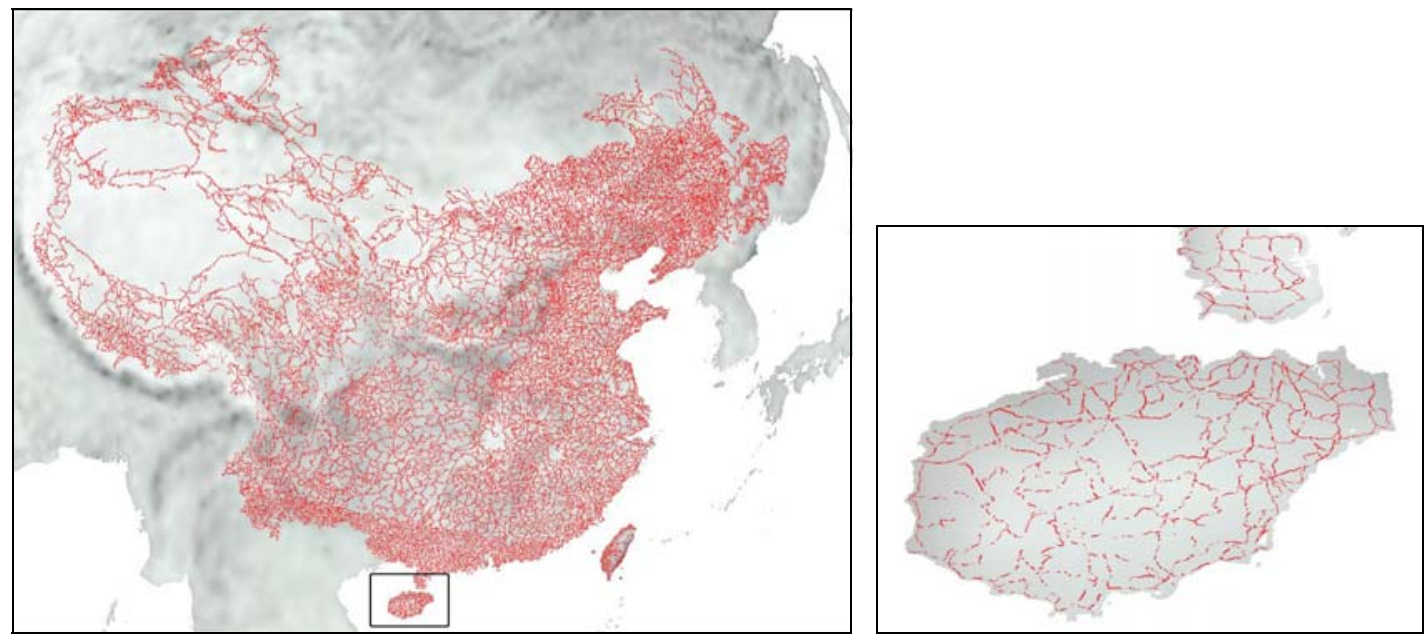

(a) Full view of China roads before drift operation (left) and local enlarge map (right).
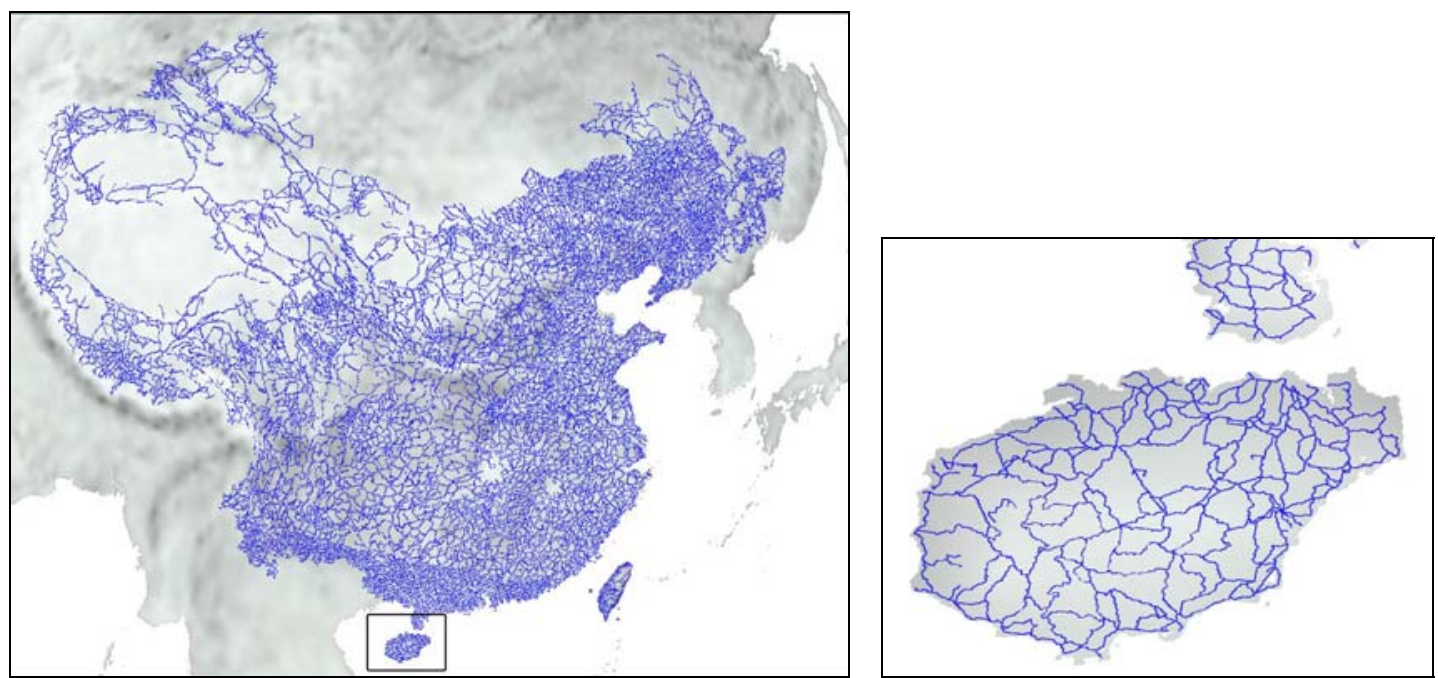

(b) Full view of China roads after drift operation (left) and local enlarge map (right).

Figure 5. Full view of China roads before (a) and after (b) drift operation (level 11).
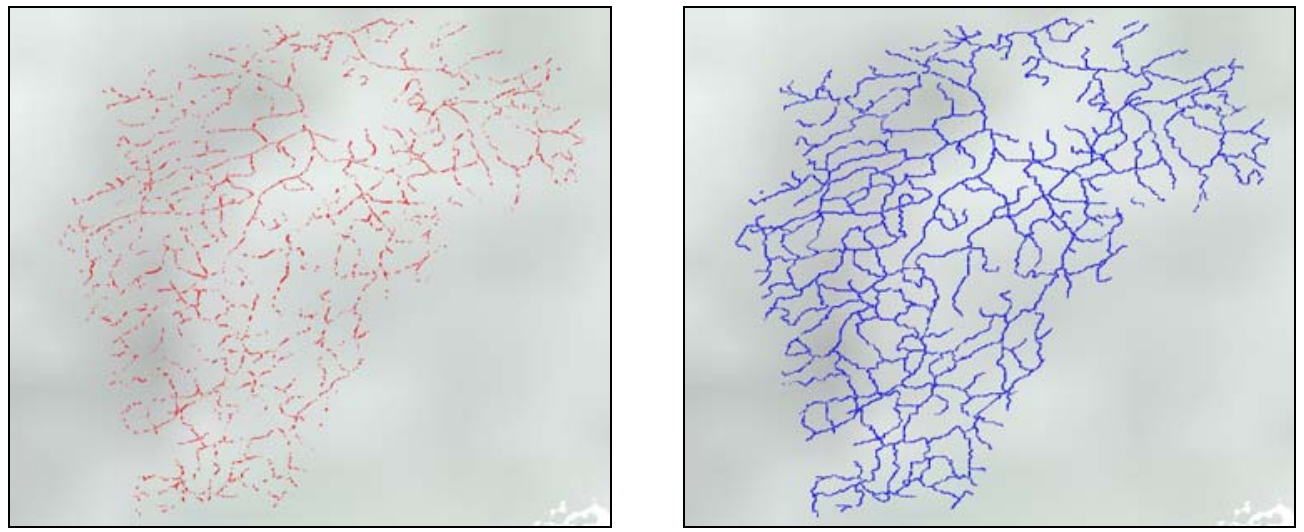

Figure 6. Full view of Jiangxi roads before (left) and after (right) shift operation (level 13) 


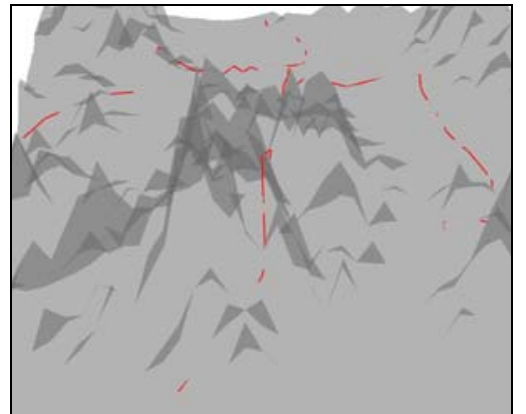

(a) Level 13

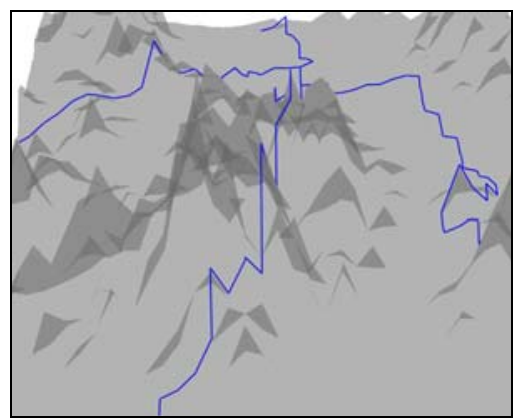

(e) Level 13-

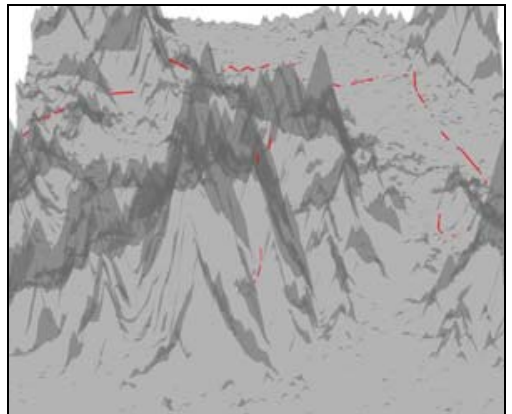

(b) Level 15

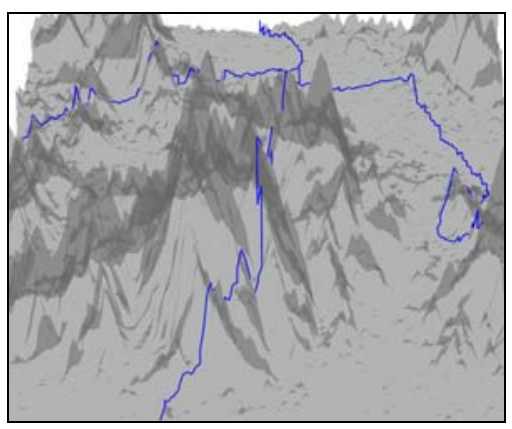

(f) Level 15-

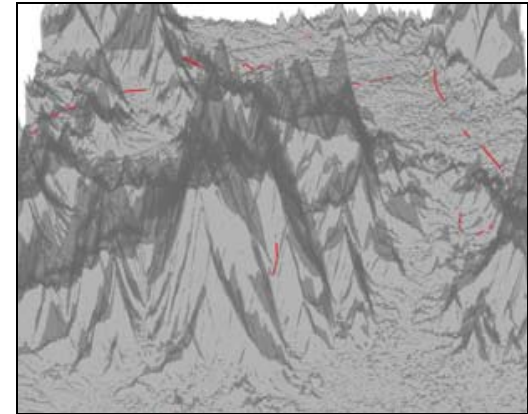

(c) Level 17

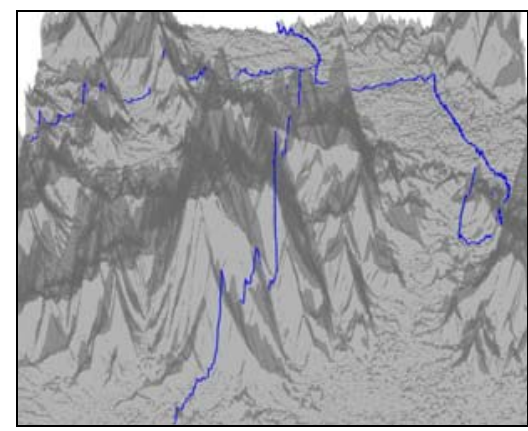

(g) Level 17-

Figure 7. Local view of Jiangxi roads before $(a, b, c)$ and after $(e, f, g)$ shift operation.

Table 1. Analysis on efficiency and error of integrating Jiangxi roads with spherical DEM

\begin{tabular}{cccccccc}
\hline Level & \multicolumn{2}{c}{ Efficiency $(\mathrm{ms})$} & \multicolumn{3}{c}{ Mean error $(\mathrm{km})$} & Average edge length of & Scale \\
\cline { 1 - 3 } & shift & interpolation & 3-D & horizontal & vertical & DQG cell $(\mathrm{km})$ & \\
\hline 13 & 2 & 9 & 0.436 & 0.324 & 0.252 & 1.391 & $\mathrm{~L}: 1000,000$ \\
14 & 4 & 18 & 0.212 & 0.159 & 0.120 & 0.696 & \\
15 & 9 & 35 & 0.113 & 0.086 & 0.063 & 0.348 & $1: 100,000$ \\
16 & 18 & 77 & 0.059 & 0.045 & 0.033 & 0.174 & \\
17 & 35 & 140 & 0.035 & 0.028 & 0.018 & 0.087 & $1: 20,000$ \\
18 & 70 & 275 & 0.025 & 0.020 & 0.012 & 0.043 & \\
\hline
\end{tabular}

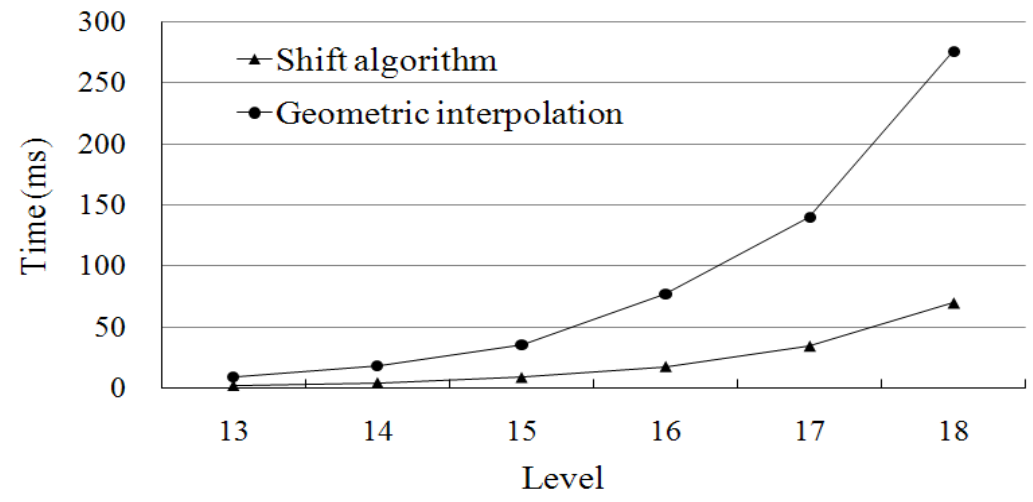

Figure 8. Comparison on efficiency of shift algorithm and geometry-based approach. 
International Archives of the Photogrammetry, Remote Sensing and Spatial Information Sciences, Volume XL-4/W2, 2013 ISPRS WebMGS 2013 \& DMGIS 2013, 11 - 12 November 2013, Xuzhou, Jiangsu, China

Topics: Global Spatial Grid \& Cloud-based Services

\section{CONCLUDING REMARKS}

To overcome the low efficiency of traditional geometry-based method for the integration of large-scale vector polyline and the terrain based on spherical grid, an adaptive 'shift algorithm' is developed to avoid the suspension and penetration and has achieved the seamless integration. The experimental result shows that the shift algorithm spends about $24 \%$ of the time consumption of the traditional method and its time consumption increases much more slowly as the subdivision level increasing. In additional, the mean error led by shift operation is totally controlled within about half a DQG cell, which entirely meets the requirements for scale accuracy and visualization.

This paper carried out preliminary construction and exploration on the adaptive integration model of vector polyline and spherical terrain at individual subdivision level. Future works will focus on dynamic update, efficient management and rendering of vector data, operation on spatial ordering, and industrial applications of spherical adaptive integration model.

\section{REFERENCES}

Bruneton E. and Neyret F,, 2008, Real-time Rendering and Editing of vector-based terrains, Eurographics, 27(2):311-320.

Döllner J., 2005, Geovisualization and Real-time 3d Computer Graphics. Exploring Geovisualization, Exploring Geovisualization, edited by Dykes J., MacEachren A. M. and Kraak M. J. (Pergamon: Elsevier Ltd.) Chapter 16, pp.325-343

Schneider M., Guthe M., and Klein R., 2005, Real-time Rendering of Complex Vector Data on 3d Terrain Models, Proc. of the 11th Int. Conf. on Virtual Systems and Multimedia (Ghent, Belgium), pp.573-582

Schneider M. and Klein R., 2007, Efficient and Accurate Rendering of Vector Data on Virtual Landscapes, Journal of WSCG, 15(1-3): 59-65.

Yang L., Zhang L. Q., and Kang Z., 2010, An Efficient Rendering Method for Large Vector Data on Large Terrain Models, Science China Information Sciences, 53(6): 1122-29(in Chinese).

Vaaraniemi M., Treib M., and Westermann R., 2011, HighQuality Cartographic Roads on High-Resolution DEMs, $19^{\text {th }}$ Int. Conf. in Central Europe on Computer Graphics, Visualization and Computer Vision (Plzen) (Vaclav Skala Union Agency), pp.41-48

Szenberg F., Gattass M., and Carvalho P., 1997, An Algorithm for the Visualization of a Terrain with Objects, Proc. of Brazilian Computer Graphic Symposium(Campos do Jordao) vol231 (Los Alamitos: IEEE Comput. Soc), pp.103-110

Agrawal A., Radhakrishna M., and Joshi R., 2006, Geometry based mapping and rendering of vector data over LOD phototextured 3D terrain models, Proc. of WSCG (Plzen) (Vaclav Skala Union Agency), pp.1-8.

Sun A., Lanig S., Neis P., and Zipf A., 2008, Integrating Terrain Surface and Street Network for 3D Routing, 3rd Int.
Workshop on 3D Geo-Info (Seoul) (Berlin:Springer) , pp.109126.

Sun W. B., Hu B. L., and Wang H. B., 2012, Integration of Vector and Terrain Data Based on Degenerated Quadtree Grid, Geography and Geo-Information Science, 28(1):43-46(in Chinese).

Cui M. J., and Zhao X. S., 2007, Tessellation and Distortion Analysis Based on Spherical DQG, Geography and GeoInformation Science, 23(6):23-25(in Chinese).

Zhou C. H., Ou Y., and Ma T., 2009, Progresses of Geographical Grid Systems Researches, Progress In Geography, 28(5):657-662(in Chinese). 